\section{Multiple Eruptive Milia Coexisting with Milia en Plaque}

A 2-month-old male presented with a 1-month history of spreading and enlarging skin lesions. Physical examination revealed multiple skin-colored papules and plaques on the dorsum of hands and feet. The plaques were composed of multiple tiny white papules (Figure 1). There was no history of trauma, including friction or rubbing, use of topical agents, and drug intake history before the development of the skin lesion. Also, his birth and family history was unremarkable. A punch biopsy was performed and histopathology revealed multiple cystic structures filled with keratin (Figure 2). Cysts were located below the epidermis and within the dermis and lined by stratified squamous epithelium. Surrounding these cysts, mild infiltration of lymphocytes was noted. Based on clinical and pathologic findings, diagnosis of milia en plaque (MEP) concomitant with multiple eruptive milia (MEM) was made and the patient is regularly being observed.

Milia are benign keratinous cysts that may cause cosmetic problems. Milia can be classified as primary or secondary [1]. Among primary milia, there are several variants, including MEM, nodular grouped milia, generalized milia with nevus depigmentosus, and MEP [1]. MEM is a rare variant of primary milia and more extensive in number and distribution than that would be expected in primary milia [2]. It is characterized by the sudden onset of milia during the course of weeks to months. MEM is most commonly seen on the head, neck and trunk. MEM may spontaneously arise without a cause, but

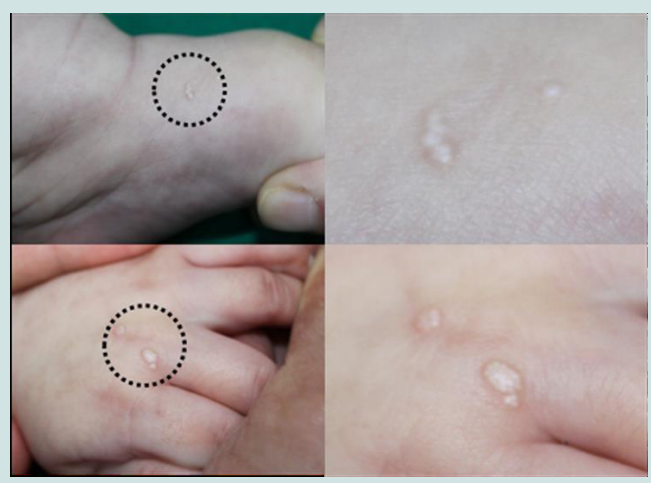

Figure 1: Clinical pictures of multiple eruptive milia and milia en plaque. Skincolored, verrucous plaques and papules on the dorsum of hands and feet.

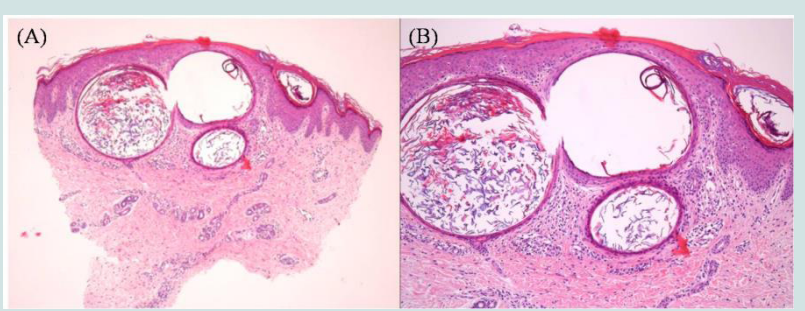

Figure 2: Histopathology revealed multiple cystic structures containing keratinous material throughout the epidermis and papillary dermis. Cyst wall is lined by two or three layers of stratified epithelium (Hematoxylin and eosin; A: $x 40, B: \times 100)$

\section{Journal of}

\section{Clinical \& Investigative} Dermatology

\author{
Yu Seok Jung, Sang Hyun Cho, Jeong Deuk Lee and \\ Hei Sung Kim* \\ Department of Dermatology, Catholic University of Korea, Incheon St. \\ Mary's Hospital, Incheon, Korea

\section{*Address for Correspondence} \\ Hei Sung Kim, Department of Dermatology, Catholic University of Korea, \\ Incheon St. Mary's Hospital, Incheon, Korea, Tel: 82-32-280-5700; \\ E-mail: hazelkimhoho@gmail.com \\ Submission: 24 October, 2016 \\ Accepted: 02 November, 2016 \\ Published: 12 November, 2016 \\ Copyright: ๑ 2016 Jung YS, et al. This is an open access article distributed \\ under the Creative Commons Attribution License, which permits unrestricted \\ use, distribution, and reproduction in any medium, provided the original work \\ is properly cited.
}

it may be inherited or may be a part of genodermatoses such as Bazex syndrome, Rombo syndrome and Gardner syndrome [2]. It is also hypothesized that sunlight or external stimuli may be associated with the pathogenesis of MEM. MEP is another clinical variant of primary milia that is characterized by numerous tiny grouped milia overlying an erythematous plaque $[3,4]$. In most previous reports, the lesions were distributed in the periauricular area $[3,4]$. The etiology of MEP remains unknown. Whereas some spontaneous regression of milia has been reported, usually the lesions persist without treatment [4]. Several therapeutic modalities have been used to treat milia including curettage, electrodessication, dermabrasion, cryotherapy, simple excision, topical retinoids, oral minocycline, photodynamic therapy and Er: YAG or $\mathrm{CO}_{2}$ laser ablation [1-4].

This case showed several unique characteristics regarding the clinical feature and distribution of lesions. Interestingly, this patient showed clinical features of MEM and MEP at the same time. Moreover, in contrast to secondary milia which commonly develop in acral lesions due to predisposing trauma or previous dermatosis, few reports has described primary milia with an acral distribution such as this case. Herein, we report a case of MEP coexisting with MEM on the hands and feet of a 2-month-old male. Increased experience with this peculiar condition will improve the awareness among dermatologists.

\section{References}

1. Jung SW, Park IH, Lee W, Seol JE, Kim H, et al. (2015) Multiple eruptive milia and milia en plaque on the hands and feet of a 2-month-old female. $J$ Dermatol 42: 931-932.

2. Cho E, Cho SH, Lee JD (2010) Idiopathic multiple eruptive milia occurred in unusual sites. Ann Dermatol 22: 465-467.

3. Barzegar M, Mozafari N (2015) A new site of milia en plaque: report of a case and review of the literature. Int J Dermatol 54: 1423-1425.

4. Stefanidou MP, Panayotides JG, Tosca AD (2002) Milia en plaque: a case report and review of the literature. Dermatol Surg 28: 291-295.

\section{Acknowledgements}

Funding Sources: This study was supported by a grant of the Korean Healthcare technology R\&D project, Ministry of Health \& Welfare, Republic of Korea (Grant No.: NH15C0105). 